\title{
Changes in the diagnosis and management of diabetes in Mexico City between 1998-2004 and 2015-19
}

\author{
Supplementary Material, Table of Contents
}

Supplementary figures

Page

1. Use of selected medications in participants aged $45-84$ years by diabetes status and cardiovascular or chronic kidney disease history at recruitment (1998-2004) and resurvey (2015-2019)

2. Mortality rates at ages $35-74$ years for each cause of death among participants with controlled and uncontrolled diabetes at recruitment

\section{Supplementary tables}

1. Characteristics of participants aged 45-84 years at recruitment in 19982004 and at resurvey in 2015-2019, overall and by diabetes status

2. Number of glucose-lowering medications reportedly taken in participants aged 45-84 years with previously-diagnosed diabetes at recruitment (1998-2004) and at resurvey (2015-2019)

3. Excess mortality at ages $35-74$ associated with controlled $(\mathrm{HbA} 1 \mathrm{c}<7 \%)$ or inadequately controlled diabetes at recruitment (1998-2004) among 135292 participants with no other chronic disease

4. Age-specific estimates of the prevalence of diabetes in Mexico and Mexico City for the years 2006 and 2017 
Webfigure 1: Use of selected medications in participants aged 45-84 years by diabetes status and cardiovascular or chronic kidney disease history at recruitment (1998-2004) and resurvey (2015-2019)

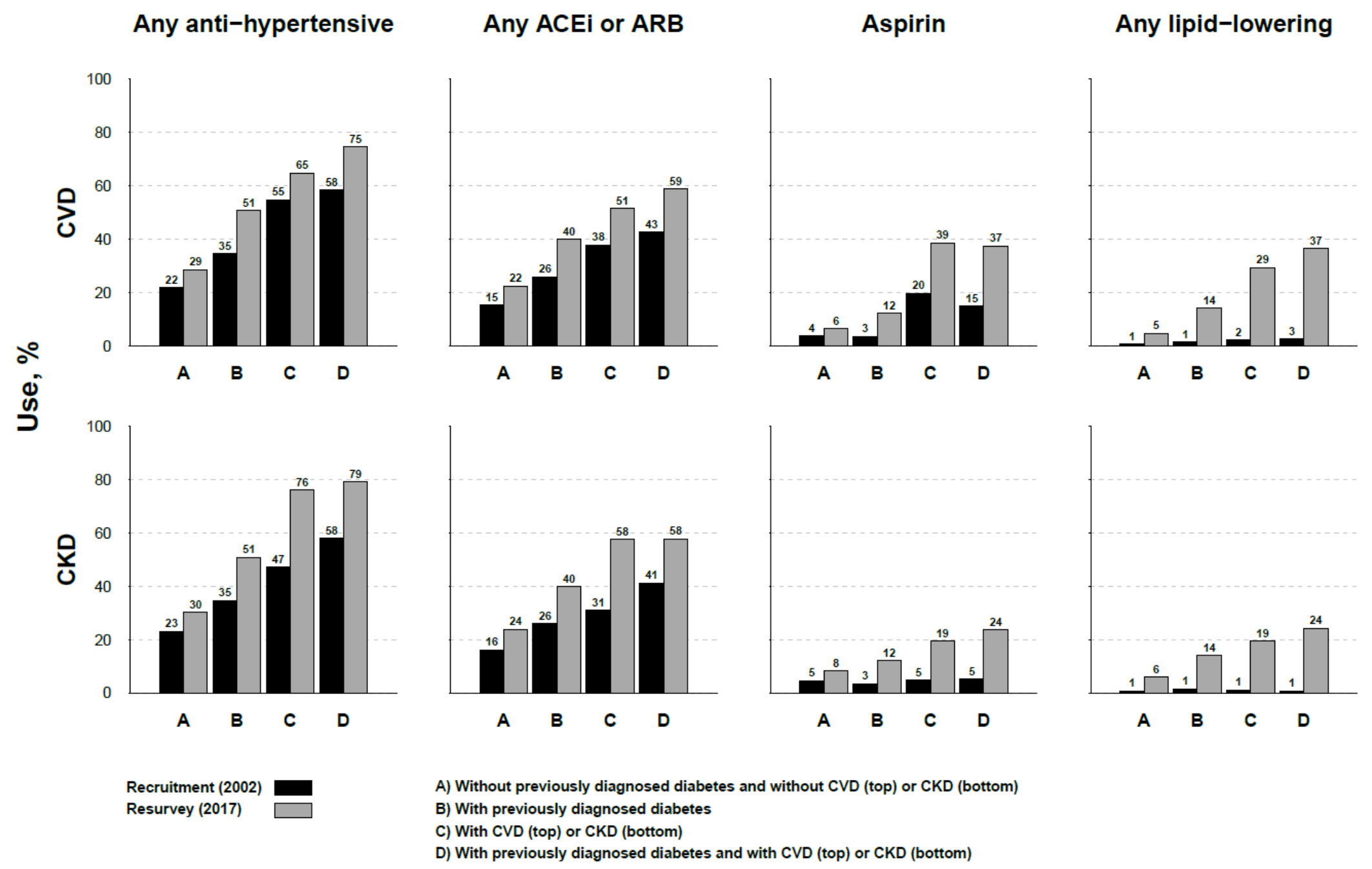

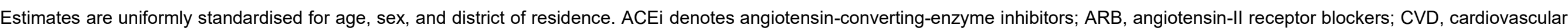

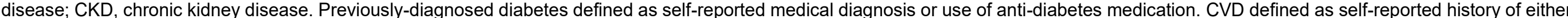

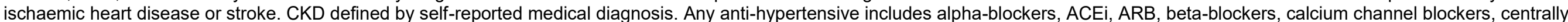
acting anti-hypertensives, and diuretics. Any lipid-lowering therapy included statins, fibrates, resins, and others. 
Webfigure 2: Mortality rates at ages 35-74 years for each cause of death among participants with controlled and inadequately controlled diabetes at recruitment

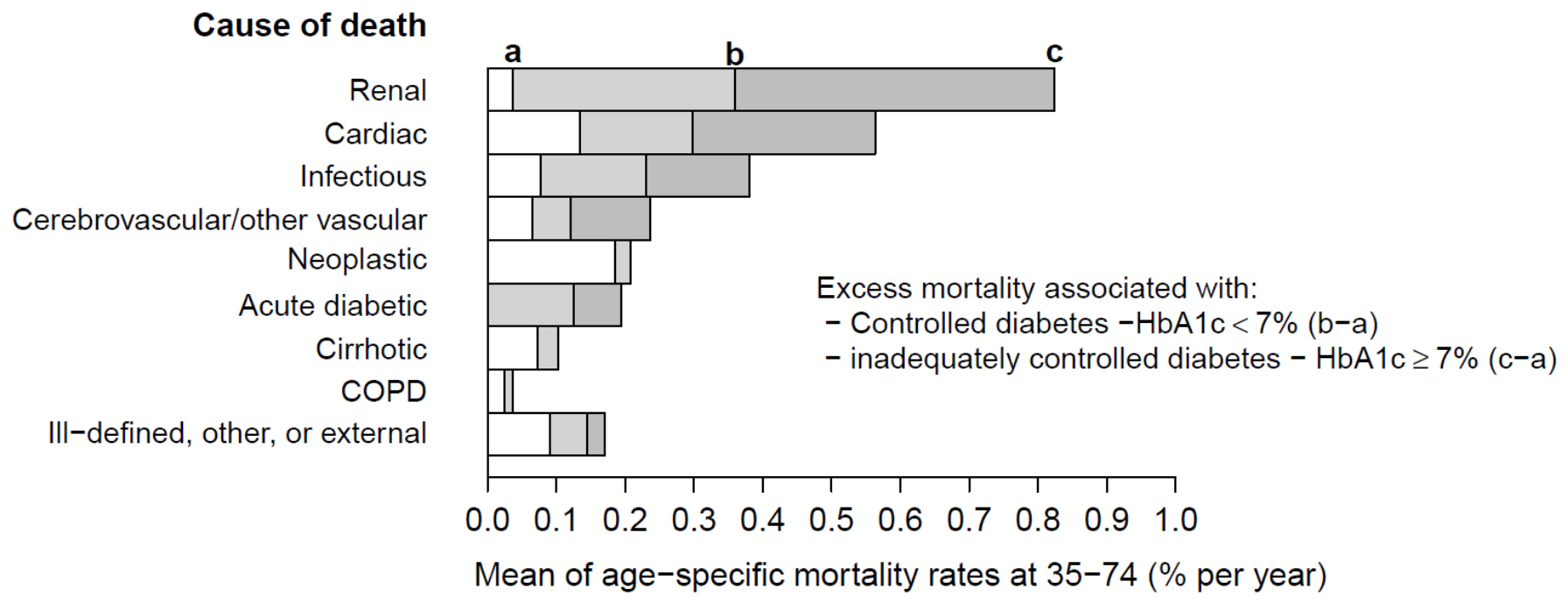

Shown are the absolute estimated disease-specific rates of death for those with inadequately controlled diabetes $\left(\mathrm{Hb} \mathrm{A}_{1 \mathrm{c}} \geq 7 \%\right.$, $\left.\mathrm{c}\right)$, controlled diabetes $\left(\mathrm{HbA}_{1 \mathrm{c}}<7 \%, \mathrm{~b}\right)$, and no diabetes at recruitment $(\mathrm{a})$. The analysis combines the percentages of participants in the current study who died between 35 and 74 years of age from particular diseases, the disease-specific rate ratios for death at 35 to 74 years of age, and 2012 national mortality rates in Mexico. The rates for all bars sum to the rates of death from any cause. The unshaded portions of the bars represent the mortality rate for the specific cause of death among participants without diabetes at recruitment. The weighted average of the death rates shown (for $16 \%$ of persons with inadequately controlled diabetes [HbA $1 \mathrm{c} \geq 7 \%$ ], $5 \%$ of persons with controlled diabetes $\left[\mathrm{HbA}_{1 \mathrm{c}}<7 \%\right.$ ], and $79 \%$ of persons without diabetes) match uniformly age-standardised 2012 Mexican national rates at ages 35 to 74 years of age for $50 \%$ men plus $50 \%$ women. 
Webtable 1: Characteristics of participants aged 45-84 years at recruitment in 1998-2004 and at resurvey in 2015-2019, overall and by diabetes status*

\begin{tabular}{|c|c|c|c|c|c|c|}
\hline & \multicolumn{3}{|c|}{ Recruitment (1998-2004) } & \multicolumn{3}{|c|}{ Resurvey (2015-2019) } \\
\hline & $\begin{array}{r}\text { No diabetes } \\
(n=74479)\end{array}$ & $\begin{array}{r}\text { Diabetes } \\
(n=25144)\end{array}$ & $\begin{array}{r}\text { Overall } \\
(n=99623)\end{array}$ & $\begin{array}{r}\text { No diabetes } \\
(n=5707)\end{array}$ & $\begin{array}{l}\text { Diabetes } \\
(n=3279)\end{array}$ & $\begin{array}{r}\text { Overall } \\
(n=8986)\end{array}$ \\
\hline Men & $34 \%$ & $33 \%$ & $34 \%$ & $30 \%$ & $28 \%$ & $30 \%$ \\
\hline \multicolumn{7}{|l|}{ Age, years } \\
\hline Mean (SD) & $58(10)$ & $61(10)$ & $59(10)$ & $65(10)$ & $67(9)$ & $66(9)$ \\
\hline $45-54$ & $46 \%$ & $31 \%$ & $42 \%$ & $16 \%$ & $9 \%$ & $13 \%$ \\
\hline $55-64$ & $27 \%$ & $33 \%$ & $29 \%$ & $35 \%$ & $34 \%$ & $34 \%$ \\
\hline $65-74$ & $18 \%$ & $26 \%$ & $20 \%$ & $29 \%$ & $36 \%$ & $31 \%$ \\
\hline $75-84$ & $9 \%$ & $11 \%$ & $9 \%$ & $20 \%$ & $22 \%$ & $21 \%$ \\
\hline $\begin{array}{l}\text { Resident of Coyoacán } \\
\text { (wealthier of the two districts) }\end{array}$ & $42 \%$ & $34 \%$ & $40 \%$ & $51 \%$ & $54 \%$ & $52 \%$ \\
\hline \multicolumn{7}{|l|}{$\begin{array}{l}\text { Demographic and lifestyle } \\
\text { characteristics }\end{array}$} \\
\hline University/college educated & $11 \%$ & $8 \%$ & $10 \%$ & $22 \%$ & $17 \%$ & $20 \%$ \\
\hline Current smoker & $24 \%$ & $22 \%$ & $23 \%$ & $18 \%$ & $16 \%$ & $18 \%$ \\
\hline \multicolumn{7}{|l|}{ Use of health services $\dagger$} \\
\hline Social security & $52 \%$ & $54 \%$ & $53 \%$ & $54 \%$ & $57 \%$ & $55 \%$ \\
\hline Other public services & $22 \%$ & $21 \%$ & $22 \%$ & $31 \%$ & $31 \%$ & $31 \%$ \\
\hline Private/Other & $26 \%$ & $25 \%$ & $26 \%$ & $15 \%$ & $11 \%$ & $13 \%$ \\
\hline $\mathrm{BMI}, \mathrm{kg} / \mathrm{m}^{2}$ & $28.4(4.6)$ & $28.8(4.8)$ & $28.5(4.7)$ & $28.2(5.1)$ & $29.1(5.7)$ & $28.4(5.3)$ \\
\hline Waist-hip ratio & $0.93(0.07)$ & $0.94(0.07)$ & $0.93(0.07)$ & $0.95(0.06)$ & $0.96(0.07)$ & $0.95(0.06)$ \\
\hline \multicolumn{7}{|l|}{ Blood pressure } \\
\hline SBP, mmHg & $133(17)$ & $136(18)$ & $134(17)$ & $130(21)$ & $136(23)$ & $132(22)$ \\
\hline $\mathrm{DBP}, \mathrm{mmHg}$ & $85(10)$ & $86(11)$ & $85(10)$ & $80(11)$ & $81(11)$ & $80(11)$ \\
\hline SBP/DBP <140/90 mmHg & $58 \%$ & $51 \%$ & $56 \%$ & $66 \%$ & $57 \%$ & $63 \%$ \\
\hline \multicolumn{7}{|l|}{ Diabetes } \\
\hline Undiagnosed $\ddagger$ & - & $24 \%$ & $6 \%$ & - & $11 \%$ & $3 \%$ \\
\hline Previously-diagnosed $\S$ & - & $76 \%$ & $20 \%$ & - & $89 \%$ & $31 \%$ \\
\hline $\mathrm{HbA}_{1 c}, \%$ & $5.5(5.3-5.8)$ & $8.2(6.8-10.2) \pi$ & $5.7(5.4-6.3)$ & $5.5(5.3-5.7)$ & $7.3(6.3-9.2) \Upsilon$ & $5.7(5.4-6.4)$ \\
\hline \multicolumn{7}{|l|}{ Self-reported comorbidities } \\
\hline Cardiovascular disease & $4 \%$ & $6 \%$ & $5 \%$ & $6 \%$ & $10 \%$ & $7 \%$ \\
\hline Coronary heart disease & $3 \%$ & $4 \%$ & $3 \%$ & $4 \%$ & $7 \%$ & $5 \%$ \\
\hline Stroke & $2 \%$ & $2 \%$ & $2 \%$ & $2 \%$ & $3 \%$ & $2 \%$ \\
\hline Chronic kidney disease & $1 \%$ & $2 \%$ & $1 \%$ & $1 \%$ & $4 \%$ & $2 \%$ \\
\hline
\end{tabular}

Mean (SD), median (IQR) or \% shown. BMI, body-mass index; SBP, systolic blood pressure; DBP, diastolic blood pressure; $\mathrm{HbA}_{1 \mathrm{c}}$, glycosylated haemoglobin.

*With the exception of age, sex and district, all estimates are uniformly standardised for age, sex and district.

† At recruitment, the question related to use of health services was added part way through recruitment; the percentages shown relate to 51,972 participants aged 45-84 years who were asked this question, all of which resided at the Iztapalapa district (at resurvey, this information was requested from all participants).

$\ddagger$ No previous diagnosis of diabetes but measured $\mathrm{HbA} 1 \mathrm{c} \geq 6.5 \%$

$\S$ Mean duration of diabetes was 10.9 (SD 8.3) years at recruitment 11.5 (SD 9.5) years at resurvey.

I Of those with diabetes, the proportion with $\mathrm{HbA} 1 \mathrm{c} \geq 10 \%$ was $28 \%$ at recruitment and $18 \%$ at resurvey. 
Webtable 2: Number of glucose-lowering medications reportedly taken in participants aged 45-84 years with previously-diagnosed diabetes at recruitment (1998-2004) and at resurvey (2015-2019)

\begin{tabular}{lccc}
\hline & \multicolumn{3}{c}{$\begin{array}{c}\text { Glucose-lowering medication use } \\
\text { (number of drugs) }\end{array}$} \\
\cline { 2 - 4 } Age, years & $\mathbf{0}$ & $\mathbf{1}$ & $\mathbf{2 2}$ \\
\hline Recruitment 2002 & & & \\
\hline $45-54$ & $22 \%$ & $62 \%$ & $15 \%$ \\
$55-64$ & $21 \%$ & $66 \%$ & $14 \%$ \\
$65-74$ & $18 \%$ & $68 \%$ & $13 \%$ \\
$75-84$ & $18 \%$ & $71 \%$ & $11 \%$ \\
Age-standardised total, 45-84 & $\mathbf{2 0 \%}$ & $\mathbf{6 7 \%}$ & $\mathbf{1 3 \%}$ \\
years & & & \\
\hline Resurvey 2017 & $8 \%$ & $50 \%$ & $42 \%$ \\
\hline $45-54$ & $9 \%$ & $49 \%$ & $43 \%$ \\
$55-64$ & $8 \%$ & $47 \%$ & $\mathbf{4 5} \%$ \\
$65-74$ & $8 \%$ & $54 \%$ & $38 \%$ \\
$75-84$ & $\mathbf{8} \%$ & $\mathbf{5 0 \%}$ & $\mathbf{4 2} \%$ \\
Age-standardised total, $45-84$ & & & \\
years & $8 \%$
\end{tabular}

Percentages standardised for district and sex. The overall estimates for ages $45-84$ are uniformly age-standardised (ie, they are the simple average of the four ten-year estimates from $45-54$ years to $75-84$ years). 
Webtable 3: Excess mortality at ages $35-74$ associated with controlled (HbA1c $<7 \%$ ) or inadequately controlled diabetes at recruitment (1998-2004) among 135292 participants with no other chronic disease

\begin{tabular}{|c|c|c|c|c|c|c|c|c|c|c|c|}
\hline \multirow[b]{2}{*}{$\begin{array}{l}\text { Age at } \\
\text { recruitment }\end{array}$} & \multicolumn{3}{|c|}{ No. participants } & \multicolumn{3}{|c|}{$\begin{array}{l}\text { No. deaths during follow-up } \\
\text { (\& before age } 75 \text { ) among those with }\end{array}$} & \multicolumn{2}{|c|}{$\begin{array}{l}\text { Mortality RR, } 95 \% \mathrm{Cl} \\
\text { (versus no diabetes) }^{*}\end{array}$} & \multicolumn{3}{|c|}{$\begin{array}{l}\text { Excess deaths associated with diabetes } \\
\text { (\% of total) }\end{array}$} \\
\hline & $\begin{array}{r}\text { Controlled } \\
\text { diabetes }\end{array}$ & $\begin{array}{r}\text { Inadequately } \\
\text { controlled } \\
\text { diabetes }\end{array}$ & $\begin{array}{r}\text { No } \\
\text { diabetes }\end{array}$ & $\begin{array}{r}\text { Controlled } \\
\text { diabetes }\end{array}$ & $\begin{array}{r}\text { Inadequately } \\
\text { controlled } \\
\text { diabetes }\end{array}$ & $\begin{array}{r}\text { No } \\
\text { diabetes }\end{array}$ & $\begin{array}{r}\text { Controlled } \\
\text { diabetes }\end{array}$ & $\begin{array}{r}\text { Inadequately } \\
\text { controlled } \\
\text { diabetes }\end{array}$ & $\begin{array}{r}\text { Controlled } \\
\text { diabetes }\end{array}$ & $\begin{array}{r}\text { Inadequately } \\
\text { controlled } \\
\text { diabetes }\end{array}$ & All diabetes \\
\hline $35-44$ & 933 & 2771 & 47,946 & 67 & 435 & 1046 & $3.0(2.3,3.9)$ & $7.0(6.2,7.8)$ & $45(3 \%)$ & $373(24 \%)$ & $418(27 \%)$ \\
\hline $45-54$ & 1729 & 5522 & 32,734 & 200 & 1171 & 1474 & $2.5(2.2,2.9)$ & $4.7(4.4,5.1)$ & $121(4 \%)$ & $924(32 \%)$ & $1045(37 \%)$ \\
\hline $55-64$ & 1994 & 5458 & 18,931 & 352 & 1516 & 1695 & $2.1(1.9,2.3)$ & $3.4(3.1,3.6)$ & $183(5 \%)$ & $1067(30 \%)$ & $1250(35 \%)$ \\
\hline $65-74$ & 1682 & 3836 & 11,756 & 214 & 605 & 690 & $2.3(2.0,2.7)$ & $2.7(2.4,3.0)$ & $121(8 \%)$ & $381(25 \%)$ & $502(33 \%)$ \\
\hline Total & 6338 & 17,587 & 111,367 & 833 & 3727 & 4905 & $2.3(2.1,2.5)$ & $3.9(3.8,4.1)$ & $470(5 \%)$ & $2745(29 \%)$ & 3215 (34\%) \\
\hline
\end{tabular}

*Age-specific mortality rate ratio estimates for those with versus without diabetes at recruitment are adjusted for sex, district, education, smoking, height, weight, waist and hip circumference. Analyses exclude those with any prior diagnosis of chronic kidney disease or ischaemic heart disease, stroke, cirrhosis, cancer or emphysema.

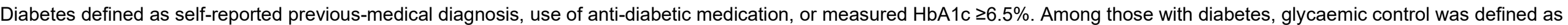
$\mathrm{HbA} 1 \mathrm{c}<7 \%$. 
Webtable 4: Age-specific estimates of the prevalence of diabetes in Mexico and Mexico City for the years 2006 and 2017

\begin{tabular}{lllll}
\hline \multirow{2}{*}{ Age, years } & \multicolumn{2}{c}{ Mexico } & \multicolumn{2}{c}{ Mexico City } \\
\cline { 2 - 5 } & $\mathbf{2 0 0 6}$ & $\mathbf{2 0 1 7}$ & $\mathbf{2 0 0 6}$ & $\mathbf{2 0 1 7}$ \\
\hline 45 to 49 & $17 \%$ & $20 \%$ & $17 \%$ & $23 \%$ \\
50 to 54 & $21 \%$ & $26 \%$ & $21 \%$ & $28 \%$ \\
55 to 59 & $25 \%$ & $30 \%$ & $25 \%$ & $32 \%$ \\
60 to 64 & $28 \%$ & $32 \%$ & $28 \%$ & $35 \%$ \\
65 to 69 & $29 \%$ & $33 \%$ & $30 \%$ & $36 \%$ \\
70 to 74 & $30 \%$ & $34 \%$ & $30 \%$ & $36 \%$ \\
75 to 79 & $30 \%$ & $34 \%$ & $30 \%$ & $36 \%$ \\
80 to 84 & $30 \%$ & $33 \%$ & $30 \%$ & $35 \%$ \\
\hline $\begin{array}{l}\text { Age standardised total, } \mathbf{4 5} \\
\text { to } 84 \text { years }\end{array}$ & $\mathbf{2 6 \%}$ & $\mathbf{3 0 \%}$ & $\mathbf{2 6 \%}$ & $\mathbf{3 3 \%}$ \\
\hline
\end{tabular}

Data obtained from the Global Burden of Disease project at http://ghdx.healthdata.org/gbd-resultstool?params=gbd-api-2017-permalink/2703d0d555dbeb6994df25d288b8ef60 (accessed on 28 May 2020) 